\title{
Treatment algorithm for chronic lateral ankle instability
}

\author{
Sandro Giannini ${ }^{1}$ \\ Alberto Ruffilli1 \\ Gherardo Pagliazzi ${ }^{1}$ \\ Antonio Mazzotti ${ }^{1}$ \\ Giulia Evangelisti² \\ Roberto Buda ${ }^{1}$ \\ Cesare Faldini ${ }^{3}$
}

1 I Clinic of Orthopaedics and Traumatology, Rizzoli Orthopaedic Institute, Bologna Italy

2 Department of General Surgery and Orthopaedics, Villa Erbosa, Bologna, Italy

3 Department of Rizzoli, Sicilia, Orthopaedic Service, Rizzoli Orthopaedic Institute, Bagheria, Italy

\author{
Corresponding author: \\ Gherardo Pagliazzi \\ I Clinic of Orthopaedics and Traumatology, Rizzoli \\ Orthopaedic Institute \\ Via G.C. Pupilli 1 \\ 40136 Bologna, Italy \\ E-mail: gherardo.pagliazzi@gmail.com
}

\section{Summary}

Introduction: ankle sprains are a common sportsrelated injury. A $20 \%$ of acute ankle sprains results in chronic ankle instability, requiring surgery. Aim of this paper is to report the results of a series of $\mathbf{3 8}$ patients treated for chronic lateral ankle instability with anatomic reconstruction. Materials and methods: thirty-eight patients were enrolled in the study. Seventeen patients underwent a surgical repair using the Brostrom-modified technique, while the remaining underwent anatomic reconstruction with autologous or allogenic graft.

Results: at a mean follow-up of 5 years the AOFAS score improved from $66.1 \pm 5.3$ to $92.2 \pm 5.6$.

Discussion: the findings of this study confirm that anatomic reconstruction is an effective procedure with satisfactory subjective and objective results which persist at long-term follow-up along with a low complication rate. No differences, in term of clinical and functional outcomes, were observed between the Brostrom-modified repair and the anatomic reconstruction technique.

Level of evidence: level IV.
KEY WORDS: ankle sprain, chronic ankle instability, anatomic reconstruction, plantaris.

\section{Introduction}

Ankle sprains are a common sports-related injury, that predominantly occurs in skeletally mature patients, with a lower incidence in children and adolescents. Ankle sprains mostly involve the lateral ligament complex following an inversion force on a plantar flexed foot. The anterior talo-fibular ligament (AT$F L$ ), the weakest and the shortest of the ligaments of the lateral complex, is involved in the majority of lateral ankle sprains, while the calcaneo-fibular ligament (CFL) and the posterior talo-fibular ligament (PTFL) are involved in $50-75 \%$, and $<10 \%$ respectively ${ }^{1}$. Predisposing factors which may cause repetitive ankle twisting episodes such as hindfoot varus, plantarflexed first ray, and cavus foot type must be considered in the diagnosis ${ }^{2,3}$. Giannini et al. in 1983 stated that $67 \%$ of lateral ankle sprains occurred in active patients with cavus foot ${ }^{4}$.

While the $80 \%$ of acute ankle sprains reach full recovery with conservative management, a $20 \%$ of acute ankle sprains develop chronic symptoms resulting in chronic ankle instability.

Furthermore most of the chronic ankle lateral ligament lesions involved the ATFL alone or the ATFL in association with the CFL, cause more complex lesions encompassing also the PTFL are usually addressed surgically as a first line treatment.

Chronic ankle instability can be distinguished into mechanical and functional. Mechanical instability is characterized by an abnormal ankle mobility, assessed clinically using manual stress application by the anterior drawer and the talar tilt tests ${ }^{5,6}$. Functional instability was firstly described by Freeman et al. $^{7}$ as a subjective feeling of the ankle giving way during either physical activity or during common activities of daily living. Tropp in 1985 described functional instability as a movement that is independent from voluntary control, even if the physiological range of motion (ROM) is not always exceeded.

Indication for surgical treatment is represented by associated mechanical and functional chronic ankle instability unresponsive to a 3 months rehabilitation program. Isolated mechanical instability without givingway episodes is not in itself an indication of surgery; hence the surgical treatment is valued when patients are not willing to accept the recurrence of spraining episodes or subjective feelings of giving-way. 
The ankle joint is a complex anatomic structure in which stability and mobility depends from correct ligamentous balancing and support. The goal of surgery is to restore the native anatomy of the injured ligaments, focusing especially on their length, direction and tightness, in order to restore the correct kinematics of the injured ankle. More than 80 different surgical procedures for the treatment of the chronically lateral unstable ankle have been described in literature ${ }^{8}$. These can be distinguished into anatomic and non-anatomic. Anatomic techniques consist of direct repair of injured ligaments with the use of local tissue, or alternatively ligaments reconstruction using a free tendon graft. The main advantages of anatomic techniques are the simplicity of the procedure, the restoration of the physiological joint anatomy and kinematics, and the preservation of the subtalar joint mobility ${ }^{9,10}$.

Non-anatomic procedures encompass tenodesis in order to substitute the injured ligaments, and to address the pathological ankle mobility. These techniques often result in joint stiffness and limited range of motion of the ankle and subtalar joints ${ }^{2,11-13}$.

Even if there is no clear consensus on which type of technique should be adopted to achieve the best results, our preferred choice is the Brostrom-modified repair using a regional periosteal flap when local tissues have good quality, or alternatively an anatomic reconstruction using the autologous tendon of plantar gracilis or an allograft (usually the peroneus brevis tendon) in presence of poor quality local tissues, in cases of failure of previous anatomic reconstructions or when a general ligamentous laxity is present. In presence of any associated foot abnormality increasing the supination force through the lateral ankle and foot, the surgeon should address it and correct it along with the ligamentous reconstruction.

Aim of this paper is to report the clinical and radiographical results of a series of 38 patients affected by chronic lateral ankle instability and treated with the described algorithm at a mean of 5 years of follow-up (range 2-8).

\section{Materials and methods}

Thirty-eight patients (25 males and 13 females with a mean age of $25.9 \pm 7.4$ years) were treated for symptomatic chronic lateral ankle instability. Inclusion criteria were patients less than 50 years old, with unilateral involvement of the lateral ligament complex with pain associated to functional and mechanical instability unresponsive to a minimum of 3 months of conservative treatment. Conservative treatment consisted in a physical therapy program focused on improvement of ankle joint proprioception, associated with active calf and peroneal muscles strengthening. Contraindications for surgery included inflammatory rheumatic disease, infections, reflex sympathetic dystrophy, osteopenia, vascular and neurologic diseases. Patients with associated medial instability, osteochondral lesions or peroneal tendon pathologies were also excluded from the study.
The ATFL was injured in all the cases treated, while in 11 patients an associated lesion of the CFL was present. Mean time elapsed from the first ankle twisting episode to surgery was 30 months (range 25-36). Seventeen patients (12 cases of isolated ruptures of the ATFL and 5 ruptures of both the ATFL and CFL) underwent a surgical repair of the injured ligaments using the Bröstrom-modified technique; In cases of isolated rupture of the ATFL, the torn ends of the ligament injured were repaired; in cases of associated injury of the CFL, the same repair performed for the ATFL was achieved. The other 21 patients (15 cases of isolated ruptures of the ATFL and 6 ruptures of both the ATFL and CFL) underwent an anatomic reconstruction using the autologous plantaris gracilis tendon (8 cases) or a peroneus brevis allograft from cadaver (13 cases). Preoperatively the presence of the plantaris gracilis tendon was assessed by means of ultrasound evaluation. One valgus calcaneal osteotomy and 2 first metatarsal osteotomy for correction of a fixed first metatarsal head plantar deformity were performed as associated procedures. Thirtyone patients practiced sport activity. In detail 28 played at a recreational level, while 3 patients were professionals. Twenty-four patients were involved in contact sports (soccer, basketball), while the remaining 7 played noncontact sports (volleyball, tennis, ballet, aerobics).

Patients were evaluated before surgery by means of physical examination focusing on the range of motion and on the anterior drawer test performed with the knee at $90^{\circ}$ of flexion and with the ankle positioned at $10^{\circ}$ of plantar flexion as stated by Kovaelski et al. ${ }^{14}$. Weight bearing radiographs were taken and an MRI aimed to confirm the ligamentous lesion and to highlight the presence of any intra-articular associated pathology was performed. The American Orthopaedic Foot and Ankle Society hindfoot (AOFAS) score was also administered to the patients ${ }^{15}$.

Patients were re-evaluated at 3, 6 and 12 months then yearly up to the mean follow-up of 5 years. The operated ankle was assessed through a physical examination focusing on the range of motion and on the anterior drawer test. The AOFAS score was also administrated to the patients. Results of the AOFAS score were rated as follows: excellent (90-100); good (70-89); fair (50-69); poor (<50).

At final follow-up weight bearing radiographs of the operated ankle were also taken to investigate the onset of arthritic changes according to the Van Dijk scale ${ }^{16}$.

Informed consent was obtained from all the patients who entered into the study and the work was approved by the Ethics Committee of Rizzoli Orthopedic Institute. The study meets the ethical standards of the Muscle, Ligaments and Tendons Journal ${ }^{17 .}$

\section{Surgical techniques}

Bröstrom-modified procedure (Fig. 1) Patients' preferred position was in semilateral decubitus, under spinal anesthesia and tourniquet control. A curved incision was made from just anterior to the distal fibula and passing inferiorly to the tip of the lat- 

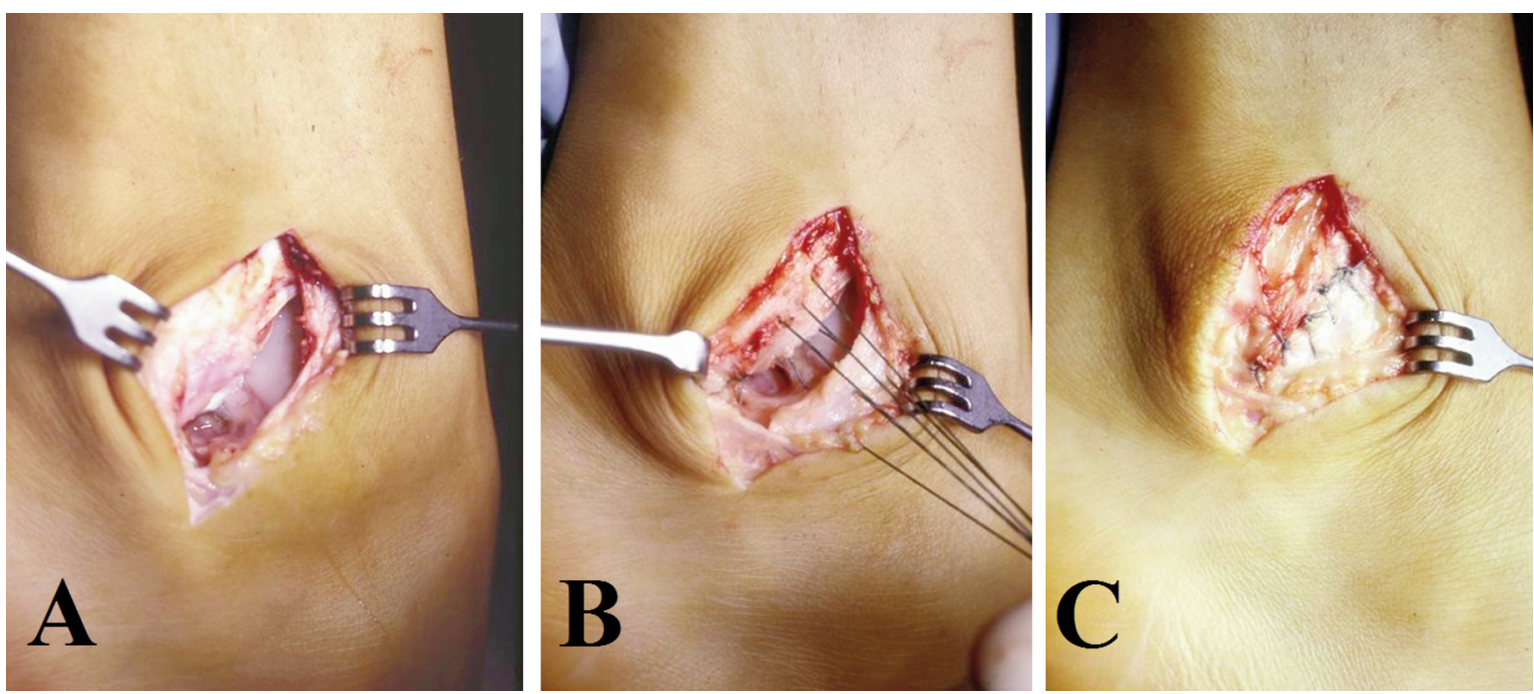

Figure 1. Surgical field showing the identification of the torn ends of the ATFL $(A)$, the reabsorbable suture threads in a transosseus fashion (B) and finally the suture of the injured ATFL with the ankle at a slight everted and dorsi flexed position (C).

eral malleolus, taking care to preserve the integrity of the superficial peroneal nerve. Alternatively a linear incision from the lateral malleolous to the sinus tarsi could be preferred. The inferior extensor retinaculum was retracted anteriorly to identify the joint capsule and the torn ends of the ATFL. A periosteal flap was elevated proximally from the distal lateral malleolus surface for about $1 \mathrm{~cm}$ long. With the ankle at a slight everted and dorsiflexed position (about 5 degrees), repair of the ATFL was performed by pulling and reattaching the distal stump of the ATFL to the lateral malleolus using reabsorbable suture threads in transosseus fashion. The periosteal flap created on the lateral malleolus was plicated in a "vest over pant" fashion over the newly sutured ATFL (Fig. 2). In order to achieve more stability and strengthening, the inferior extensor retinaculum, previously detached for the capsule, was advanced proximally and sutured to the anterior border of the lateral malleolus (Gould modification). In case of reconstruction of the CFL the inci-

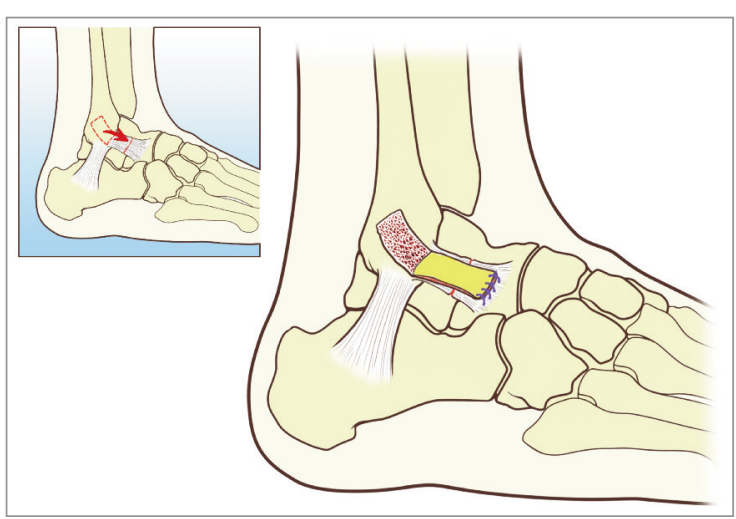

Figure 2. Schematic drawing representing the anatomic repair of the ATFL according to Brostrom and the periosteal flap plicated in a "vest over pant" fashion. sion was extended distally and posteriorly while preserving the sural nerve and the lesser saphenous vein. Then the peroneus brevis tendon was retracted in order to identify the CFL. Repair of the CFL was performed with the ankle in neutral position, by pulling proximally the distal stump of the torn CFL, and fixing it to the lateral malleolus using the same reabsorbable suture threads, as close to the anatomical origin of the CFL as possible.

\section{Anatomic reconstruction using the plantaris gracilis tendon or allograft (Fig. 3)}

Patients' preparation and position was the same described for the Bröstrom procedure.

The plantaris gracilis tendon was harvested at the ankle joint level just medial to the Achilles tendon with a 3-cm skin incision. Commonly, adhesions with the Achilles tendon near the calcaneal tuberosity or with the intermuscular fascia, made the identification of the plantaris tendon challenging. After isolation of the plantaris gracilis tendon, it was harvested using an open tendon stripper and maintaining its calcaneal insertion (Fig. 4). The graft was then tacked in its free portion with non reabsorbable suture threads. The harvested tendon was passed into the heel, throughout a drill-hole, from medial to lateral. The tendon was then passed beneath the peroneal tendons and routed into a drill-hole made into the distal fibula from posterior to anterior (Fig. 5). A tunnel was drilled in the talus at the former site of ligament insertion from lateral to medial. The plantaris gracilis tendon was inserted in the tunnel (Fig. 6) and the free tacked portion of the graft exiting on the medial side of the talus was fixed by tenodesis using non-reabsorbable stitches. In cases of absence of the plantar gracilis tendon (about 20$30 \%$ of cases in the Caucasian population), a peroneus brevis allograft was used. A 1-5 cm skin incision was made just medial to the Achilles tendon at its calcaneal insertion. The graft was fixed on the medial 

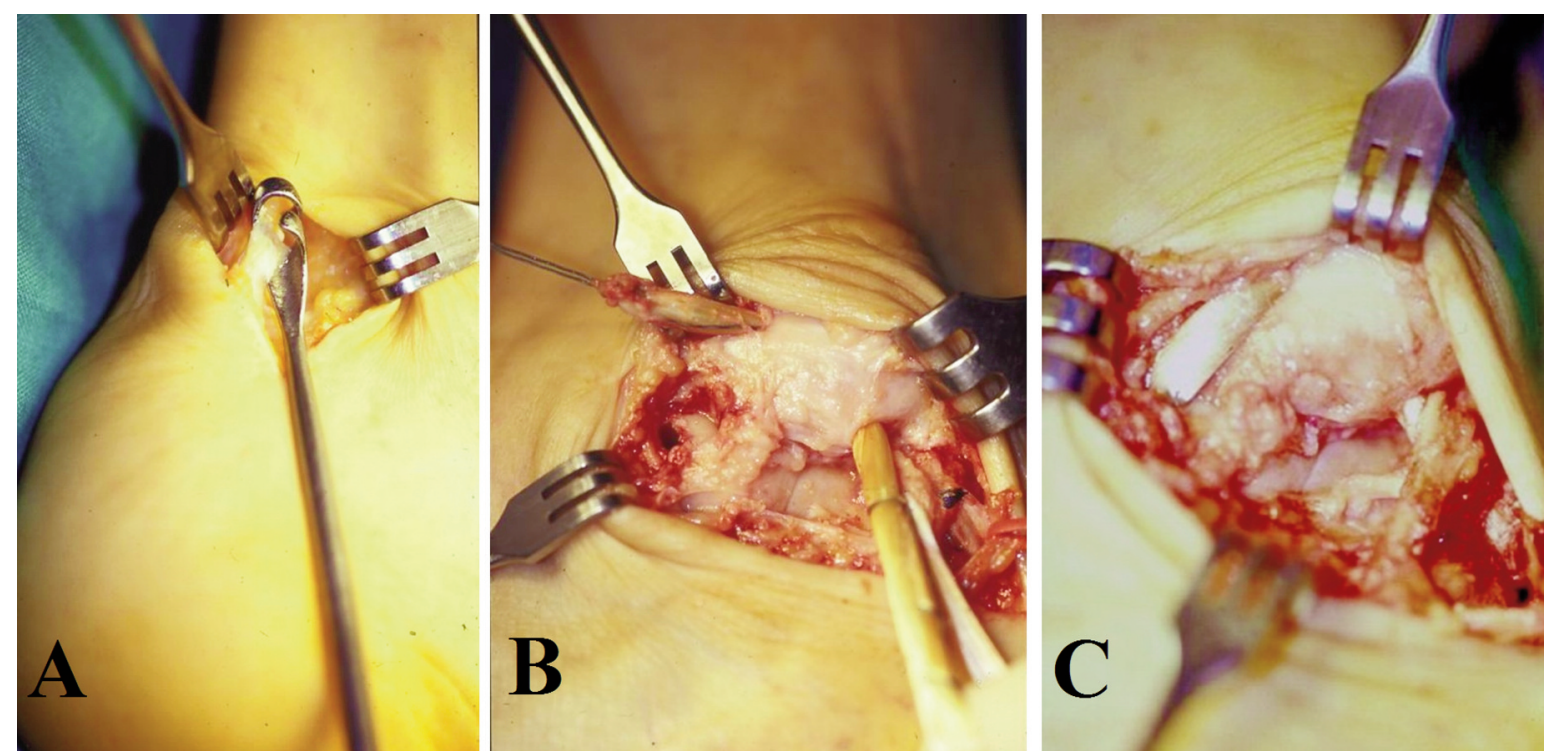

Figure 3. Surgical field showing the harvesting of the plantaris gracilis tendon $(A)$, the tendon inserted through the calcaneal tunnel and then in the malleolar hole replicating the CFL $(B)$ and finally the tendon secured throughout a drill hole in the talus reconstructing the ATFL $(\mathrm{C})$.

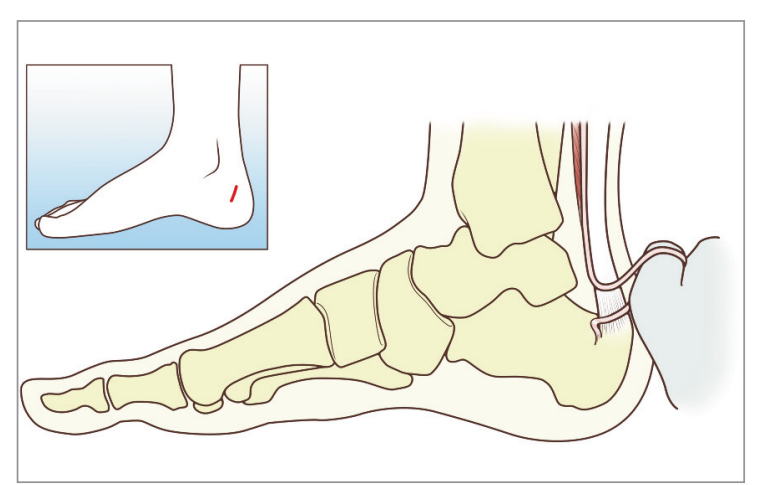

Figure 4. Schematic drawing representing the harvesting of the plantaris gracilis tendon.

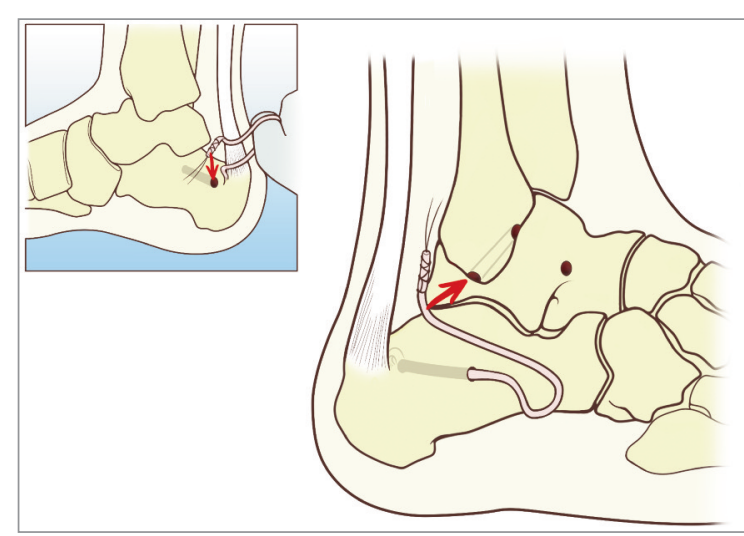

Figure 5. Schematic drawing representing graft passage through the calcaneal tunnel from medial to lateral and graft insertion inside the fibular tunnel.

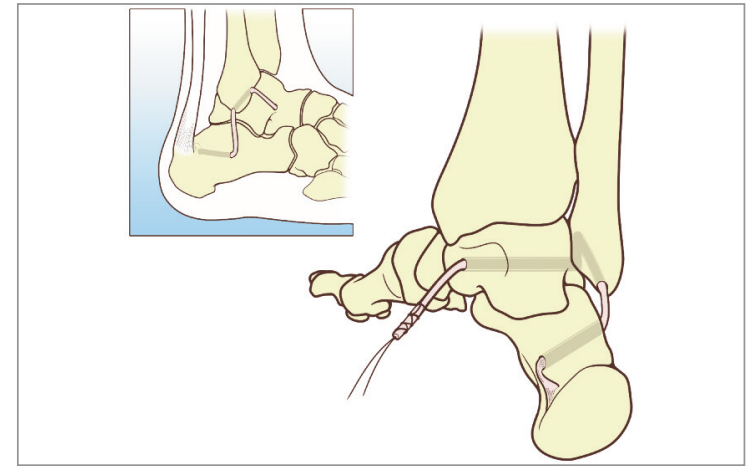

Figure 6. Schematic drawing showing the reconstructed CFL and ATFL.

border of the calcaneus using non-reabsorbable stiches or alternatively an interference screw (the interference screw was used only in the 15 cases of isolated reconstruction of the ATFL). The subsequent surgical steps were the same described for the plantaris gracilis technique.

The described technique addressed the reconstruction of both the ATFL and CFL. In cases of isolated injury of the ATFL, the plantaris gracilis tendon was detached from its calcaneal insertion. The graft was passed through the fibular tunnel directly into the talar one sited at the level of talar anatomic insertion of ATFL. Fixation on the fibular site was achieved with an anchoring device or an interference screw while the fixation on the medial talar site is achieved by tenodesis. Again in case of absence of the plantaris gracilis tendon an homologous tendon was adopted. 


\section{Post-operative treatment}

Following surgery, a non weightbearing leg brace in neutral position was advised for 2 weeks. Cautious range of motion for dorsal and plantar flexion was allowed after 2 weeks, with isometric training of all lower leg and calf muscles. From the 2 nd to the 4 th week a leg brace with $20^{\circ}$ degrees of mobility was applied, allowing partial weightbearing. After 4 weeks, the brace was removed and patients were encouraged to actively and passively move their ankle and progressive weightbearing was advised. Six-eight weeks postoperatively all patients were allowed to fully weightbear and were continued on the range of motion exercises, ankle muscles strengthening as well as proprioception training, until satisfactory recovery was achieved.

\section{Results}

No intraoperative complications were reported in this series; postoperatively there was only one case of skin incision healing delay.

At the final follow-up all the patients declared to be satisfied with the procedure. At physical examination, no signs of local tenderness on the lateral aspect of the ankle or residual swelling were detected; only 2 patients required additional physical therapies for 18 months after surgery for residual pain and discomfort on the lateral side on the ankle during sport activities. The AOFAS score improved from a preoperative value of $66.1 \pm 5.3$ to a post-operative value of $92.2 \pm$ 5.6 at final follow-up $(p<0.05)$ with 25 cases $(66 \%)$ rated as excellent and the other $13(34 \%)$ rated as good at final follow-up. In particular, AOFAS score improved to $91.8 \pm 5.2$ in the subgroup of patients treated with the Brostrom-modified technique, while, in the subgroup of patients treated with the anatomic reconstruction, the final AOFS was $92.5 \pm 6.3$. The anterior drawer test of the affected ankle showed no difference compared to the non-injured side in 26 patients, while a side-to-side difference of less than 3 $\mathrm{mm}$ was found in 12 patients.

The range of motion on the sagittal plane (dorsal and plantar flexion) of the affected ankle showed no difference compared to the non-injured ankle in 30 patients. A mean difference of less than 10 degrees was detected in 7 patients ( 3 in the subgroup of patients treated with the Bröstrom technique and 4 in the subgroup of patients treated with anatomic reconstruction), while only 1 patient (in the subgroup of patients treated with the anatomic reconstruction using the plantar gracilis tendon), reported a side-to-side difference of more than 10 degrees in plantar flexion with occasional lateral joint tenderness during strenuous sport activities.

Among the 31 sport active patients, 26 (85\%) resumed sport activities at pre-injury level after a mean of 6 months (range 4-8 months), while the remaining 5 patients $(15 \%)$ reported a return to lower-demanding sport activities. The 3 professional players were all able to resume their previous activity.
According to the Van Dijk radiographic scale (16) 30 patients $(79 \%)$ were scored as grade 0 , while the other 8 patients $(21 \%)$ were scored as grade 1 . No differences were observed with respect to the preoperative radiographs evaluated with the same scale.

\section{Discussion}

Ankle sprains represent a common occurrence during activities of daily living and sports, and lateral ligamentous complex is the most frequently injured. Unfortunately, the $20 \%$ of acute lateral ligamentous injuries hesitates in chronic instability, thus requiring surgical management $6,7,18$.

A correct diagnosis encompassing a careful patient's clinical history, a detailed clinical examination and imaging analysis is mandatory to exclude associated causes of ankle pain, such as chondral injuries, bone bruising, fractures, peroneal tendon tears and impingement syndrome. Predisposing factors which may cause or maintain instability must also be considered, focusing especially on the coexistence of a hindfoot varus and a plantar flexed first metatarsal head. These factors produce early operative failure if not corrected at the time of surgery.

Numerous anatomic and non-anatomic procedures have been described in the management of chronic ankle instability. Several studies reported an increased rate of complications in non-anatomic techniques as compared with anatomic procedures. Abnormal ankle kinematics and restricted subtalar motion are the major drawbacks encountered with nonanatomic reconstruction $3,19,20$.

Stiffness is another complication encountered more frequently with non-anatomic tenodesis $21,22$.

For these reasons the Authors decided to adopt an anatomic reconstruction in every case of lateral ligaments reconstruction in order to restore the isometricity of the torn ligaments thus obtaining an ankle kinematics as similar as possible to the controlateral healthy side.

The Bröstrom-modified technique was chosen in cases of good quality of the local tissues while the anatomic reconstruction using the plantaris gracilis tendon or allograft, was adopted in presence of weak tissues, in cases of failure of previous anatomic reconstructions or when a general ligamentous laxity was present ${ }^{23-25}$. No differences in terms of clinical outcomes were observed between the Bröstrom-modified repair and the anatomic reconstruction technique. The results obtained in our series were extremely satisfactory with 25 cases (66\%) rated excellent and 13 patients $(34 \%)$ rated good according to the AOFAS score. Joint stability investigated with the anterior drawer test proved to be normal in 26 cases and nearly normal (less than $3 \mathrm{~mm}$ of translation) in the remaining 12. A light joint stiffness, less than 10 degrees of movement, was advised only in 7 patients (3 in the subgroup of patients treated with the Brostrom technique and 4 in the subgroup of patients treated with anatomic reconstruction). The only case that complained a limitation of more than 10 degrees in 
plantar-flexion treated with anatomic reconstruction using the plantar gracilis tendon, was anyhow able to resume sport activity.

At final follow-up we didn't observe any case of recurrence of instability even if the delayed failure due to the insufficient strength of the reconstruction is the major drawback of anatomic repair techniques described in literature 24,25 .

Return to sports was highly satisfactory in this series both in recreational athletes and in professional players.

Another finding that emerged from the study is that we didn't observe arthritic changes onset at 5 years follow-up evaluating the radiographs according to the Van Dijk scale ${ }^{16}$.

We are aware that this study has some strong limitations. The number of subjects evaluated is quite low and the lack of a control group operated by non anatomic reconstruction is another factor which affects the quality of our research. In addition, the lack of the talar tilt test evaluation and the absence of ROM values in eversion/inversion are another major limitation.

Further prospective comparative studies should be performed in order to confirm the findings of the present study and the effectiveness of the described procedures. In conclusion the findings of this study confirm that anatomic reconstruction is an effective procedure with satisfactory subjective and objective results which persist at long-term follow-up along with a low complication rate. The techniques described proved to be efficient also in young and active patients involved in high demanding sports activities. Attention must be paid to address and correct any associated foot abnormalities that can increase the supination forces through the lateral ankle, causing higher stresses on the reconstructed ligaments which may result in laxity recurrence.

\section{References}

1. Amendola A, Drosdewech D. Tendon and ligament disorders of the foot and ankle, in: C. Bulstrode (Ed.) et al., Oxford textbook of orthopaedics and trauma, Vol. 2 Oxford University Press, New York. 2002;1305-1314.

2. Lee MS, Hofbauer MH. Evaluation and management of lateral ankle injuries. Clin Podiatr Med Surg. 1999;16:659-678.

3. Sammarco VJ. Complications of lateral ankle ligament reconstruction. Clin Orthop Relat Res. 2001;391:123-132.

4. Giannini S, Girolami M, Ceccarelli F, Bertelli R. Nostri orientamenti sul trattamento degli esiti delle fratture-lussazioni della Lisfranc. Chir del piede. 1983;17:169-173.

5. Safran MR, Bendetti RS, Bartolozzi III AR, Mandelbaum BR. Lateral ankle sprains: a comprehensive review part 1: etiology, pathoanatomy, istopathogenesis, and diagnosis. Med Sci Sports. 1999;31(7):S429-437.
6. Guillo S, Bauer T, Lee JW, et al. (2013) Consensus in chronic ankle instability: aetiology, assessment, surgical indications and place for arthroscopy. Orthop Traumatol Surg Res. 2013;99 (8 Suppl):S411-419.

7. Freeman MA. Instability of the foot after injuries to the lateral ligament of the ankle. J Bone Joint Surg Br. 1965;47:669-677.

8. Bauer GR. New method for reconstruction in lateral ankle instability. Jam Podiatr Med Assoc. 1995;85:459-463.

9. Bröstrom L. Sprained ankles VI. Surgical treatment of "chronic" ligament ruptures. Acta Chir Scand. 1966;132:551-565.

10. Maffulli N, Del Buono A, Maffulli GD, et al. Isolated anterior talofibular ligament Broström repair for chronic lateral ankle instability: 9-year follow-up. Am J Sports Med. 2013;41(4):858864.

11. Coughlin MJ, Schenck RC, Grebing BR, Gehron T. Comprehensive reconstruction of the lateral ankle for chronic instability using a free gracilis graft. Foot Ankle Int. 2004;25:231-241.

12. Pagenstert G, Valderrabano V, Hintermann B. Lateral ankle ligament reconstruction with free plantaris tendon graft. Tech. Foot Ankle Surg. 2005;4:104-112.

13. Liu SH, Baker CL. Comparison of lateral ankle ligamentous reconstruction procedures. Am J Sports Med. 1994;22:313-317.

14. Kovaleski JE, Norrell PM, Heitman RJ, Hollis JM, Pearsall AW. Knee and Ankle sition, Anterior Drawer Laxity, and Stiffness of the Ankle Complex. J Athl Train. 2008;43(3): 242-248.

15. Kitaoka HB, Alexander IJ, Adelaar RS, Nunley JA, Myerson MS, Sanders M. Clinical rating systems for the ankle-hindfoot, midfoot, hallux, and lesser toes. Foot Ankle Int. 1994;15(7): 349-353.

16. Van Dijk CN, Verhagen RA, Tol JL. Arthroscopy for problems after ankle fracture. J Bone Joint Surg Br. 1997;79(2):280-284.

17. Padulo J, Oliva F, Frizziero A, Maffulli N. Muscles, Ligaments and Tendons Journal. Basic principles and recommendations in clinical and field science research. MLTJ. 2013;4:250-252.

18. Chan KW, Ding BC, Mroczek KJ. Acute and chronic lateral ankle instability in the athlete. Bull NYU Hosp Jt Dis. 2011;69 (1):17-26.

19. Becker HP, Ebner S, Ebner D, et al. 12-year outcome after modified Watson-Jones tenodesis for ankle instability. Clin Orthop Relat Res. 1999;(358):194-204.

20. Hoy GA, Henderson IJ. Results of Watson-Jones ankle reconstruction for instability. The influence of articular damage. J Bone Joint Surg Br. 1994;76(4):610-613.

21. Krips R, Van Dijk N, Halasi T, et al. Long-term outcome of anatomical reconstruction versus tenodesis for the treatment of chronic anterolateral instability of the ankle joint: a multicenter study. Foot Ankle Int. 2001;22:415-421.

22. Sugimoto $\mathrm{K}$, Takakura $\mathrm{Y}$, Akiyama K, et al. Long-term results of Watson-Jones tenodesis of the ankle. Clinical and radiographic findings after ten to eighteen years of follow-up. J. Bone Joint Surg. 1998;80-A:1587-1596.

23. Caprio A, Oliva F, Treia F, Maffulli N. Reconstruction of the lateral ankle ligaments with allograft in patients with chronic ankle instability. Foot Ankle Clin. 2006;11(3):597-605.

24. Ellis SJ, Williams BR, Pavlov H, Deland J. Results of anatomic lateral ankle ligament reconstruction with tendon allograft. HSS J. 2011;7(2):134-140.

25. Colville MR. Surgical treatment of the unstable ankle. J Am Acad Orthop Surg. 1998;6:368-377. 